\title{
Calidad del control glicémico según la hemoglobina glicosilada vs la glicemia en ayunas: Análisis en una población urbana y otra rural de diabéticos costarricenses
}

Adriana Laclé-Murray ${ }^{1}$, Manuel Francisco Jiménez-N avarrete²,

1. Instituto de Investigaciones en Salud (INISA), Universidad de Costa Rica.

2. Programa de Pacientes Crónicos, Hospital de La Anexión,N icoya.

Abreviaturas: DM, diabetes mellitus; HbAlc, hemoglobina glicosilada fracción A 1c; EBAIS, Equipo (s) Básico (s) de A tención Integral en Salud; ADA,A sociación Americana de Diabetes, por sus siglas en inglés); ALAD (Asociación Latinoamericana de Diabetes); C C SS, Caja Costarricense de Seguro Social; $\mathrm{mg} / \mathrm{dl}$,miligramos por decilitro; U, diabéticos urbanos; $R$, diabéticos rurales.

ISSN 0001-6002/2004/46/3/139-144 Acta Médica Costarricense,@2004 Colegio de Médicos y Cirujanos

\begin{abstract}
Resumen
La hemoglobina glicosilada (HbA1c) es actualmente la mejor prueba disponible que refleja el control glicémico del paciente diabético, pero su uso y disponibilidad no están ordenados en el sistema nacional de salud de Costa Rica, aunque sí normados en las "Guías de atención al paciente diabético". El objetivo de este estudio fue comparar la calidad del control glicémico, según se use la glicemia en ayunas o el nivel de HbA1c en pacientes diabéticos costarricenses, de una comunidad urbana y otra rural.
\end{abstract}

Materiales y métodos: Estudio retrospectivo descriptivo, que documenta los dos parámetros mencionados en 237 diabéticos, controlados en 6 EBAIS urbanos del área de Salud Desamparados 3, provincia de San José, y en 257 diabéticos controlados en 3 EBAIS rurales del área de Salud de Nicoya, provincia de Guanacaste, durante el año 2000. En los pacientes de Desamparados se registró la glicemia más próxima al mes de julio de 2000, y su respectiva HbA1c tomada concomitantemente. En los diabéticos nicoyanos se recolectaron todas las glicemias del año 2000 y sus respectivas $\mathrm{HbA1c}$, con un promedio anual de cada una de las variables.

Resultados: Los niveles de glicemia de los diabéticos urbanos (U) se presentaron con mayor frecuencia en los rangos superiores: $>200 \mathrm{mg} / \mathrm{dl}$ en un $35 \%$; de $141-180 \mathrm{mg} / \mathrm{dl}$, un $20.5 \%$, y solo el $17.9 \%$ estaba en rangos normales de $60-110 \mathrm{mg} / \mathrm{dl}$, como muestra de un pobre control glicémico. Los diabéticos de zona rural (R) indicaron niveles similares, aunque levemente mejor controlados, con $>200 \mathrm{mg} / \mathrm{dl}$ en un $27 \%$, de $141-180$, un $22.9 \%$, y de $60-110$, un $22.3 \%$. La distribución porcentual, según niveles de HbA1c en la zona urbana, fue: $>9.5 \%$ un $34 \%$, entre el $8 \%$ y el $9.5 \%$ un $22.8 \%$, y solo el $21.1 \%$ fue menor al $6.5 \%$, mientras que los de zona rural se ubicaron con porcentajes del $40.4 \%$, el $24.1 \%$ y el $14.0 \%$, respectivamente.

$\mathrm{Al}$ relacionar los niveles de glicemia con los rangos de equivalencia de $\mathrm{HbA1c}$, se encontró que en el rango de $60-110 \mathrm{mg} / \mathrm{dl}$ solo el $58.5 \%$ de la zona urbana y el $65.3 \%$ de la zona rural, estaban con HbAlc $<6.5 \%$; un $22 \%$ en zona urbana y un $10.5 \%$ en la rural tenían niveles superiores al $8 \%$. Para el rango de 110-126 mg/dl el 30\% urbano y el 37.4\% rural tenían Hba1c > 8\%. Los rangos superiores de glicemia (>200mg/dl) sí presentaron buena correlación con Hba1c > 9.5 del 75.6\% en urbanos y el $82.9 \%$ en rurales.

Conclusión: El estudio demostró que es necesario realizar la HbA1c para valorar la calidad del control metabólico, sobre todo en pacientes que manejan glicemias en ayunas con valores $<180 \mathrm{mg} / \mathrm{dl}$. Alrededor de un $30 \%$ de los pacientes con niveles de glicemia considerados buenos en ayunas, tenían su HbA1c en un nivel malo o crítico. Por sí sola, la glicemia en ayunas no reveló el verdadero estado del control glicémico, por lo que la disponibilidad de HbA1c debe garantizarse en todas las áreas de salud de Costa Rica, para su uso cada 3 meses, como se especifica en las normas de la Seguridad Social.

Descriptores: Diabetes mellitus, hemoglobina glicosilada, control glicémico, glicemia en ayunas.

Recibido: 1 de octubre de 2003

Aceptado: 04 de mayo de 2004 
La Diabetes Mellitus (DM) es una de las pandemias más importantes de la actualidad, y la tipo dos en particular supone hoy un problema sanitario y socioeconómico de primera magnitud ${ }^{1}$.

En los Estados Unidos de Norteamérica, por ejemplo, aproximadamente 15.7 millones de personas presentan diabetes, un tercio de ellas no diagnosticadas, con un promedio de 727000 nuevos casos anuales, y una prevalencia estimada de diabetes diagnosticada en adultos, de 2900 a 3400 por cien mil ${ }^{2}$.

Desde la implementación del Plan de Reforma del Sector Salud en $1994^{3}$, Costa Rica se subdividió en tres niveles de atención en salud. El primer nivel de atención (atención primaria) se subdividió en áreas de salud (sinónimo de cantones) que a su vez se subdividieron en sectores, cada uno atendido por uno o más equipos básicos de atención integral en salud (EBAIS), según la densidad poblacional (4000 personas por EBAIS). Esto ha permitido una cobertura alta de atención en todo el territorio nacional.

Aún con esta infraestructura, no se conoce la prevalencia real de la DM tipo 2, la cual se estima entre un 5-6\% de la población mayor de 20 años, pero sí se conoce bien el impacto de sus complicaciones crónicas. En Costa Rica desde hace una década se conoce que la DM es la novena causa específica de muerte, la octava causa de consulta en hombres y la cuarta en mujeres, y es la cuarta causa de hospitalizaciones ${ }^{4}$. También ocupa el primer lugar en costos hospitalarios del país.

Existe evidencia científica que correlaciona las complicaciones a largo plazo con los niveles elevados de HbA1c y el pobre control de la DM. El ligamen que existe entre la hiperglicemia persistente y el riesgo de complicaciones microvasculares está bien establecido en varios estudios ${ }^{5,9}$.

La HbA1c es la mejor prueba disponible que refleja el control glicémico del paciente diabético10. Esta prueba ha permitido estratificar a los pacientes en categorías de riesgo para desarrollar complicaciones microvasculares, por lo que sirve para evaluar y pronosticar el futuro de los pacientes. La HbA1c puede ayudar a intensificar a tiempo la terapia de control de la DM (control glicémico), así como a identificar los casos que requieran atención especial (enfoque de riesgo).

En Costa Rica, algunos estudios en determinadas comunidades han demostrado que existe un pobre control del diabético ${ }^{11,13}$. Su control glicémico se ha medido sustancialmente por la glicemia en ayunas, prueba que refieren el nivel puntual de azúcar en la sangre y está sujeta a muchas variables, como la dieta de los días anteriores, enfermedades agudas, estado de hidratación, etc. A pesar de no ser esta prueba la más apta para valorar el control glicémico, no es hasta hace pocos años que se le ha comenzado a dar importancia a la HbA1c en la Caja Costarricense de Seguro Social (CCSS).
Lamentablemente su uso ha predominado a nivel hospitalario, en donde la mayoría de los pacientes ya tienen las complicaciones crónicas de la DM, por lo que un monitoreo de control con esta prueba, aunque es de utilidad, no produce el beneficio de prevención secundaria que podría darse en los diabéticos que se manejan en atención primaria.

Este estudio pretende comparar la evaluación del control glicémico, según se usen las glicemias en ayunas o los niveles de Hb1Ac de pacientes diabéticos de una comunidad urbana y otra rural, manejados principalmente en atención primaria.

\section{Metodología}

Población: El área de salud Desamparados se encuentra en la provincia de San José, en el centro del país, es parte de la capital y es urbana, mientras que el área de salud de Nicoya se ubica al noroeste, en la provincia de Guanacaste, separada por 400 kilómetros de la de Desamparados, en una región rural. Ambas poseen condiciones socioeconómicas y de accesibilidad a servicios de salud muy diferentes ${ }^{14}$. En estas dos áreas de salud se trabajó con:

- 443 pacientes diabéticos controlados en 3 EBAIS (Suroeste, Noreste, y Barrio San Martín) del área de Salud de Nicoya, durante el año 2000.

- 572 pacientes diabéticos registrados en el año 2000 en 6 EBAIS del área de Salud de Desamparados 3 (Fátima, Patarrá, Guidos 2 y 4, Río Azul y Linda Vista).

La muestra para el análisis consistió en aquellos pacientes que tenían al menos una determinación de hemoglobina glicosilada en ese año: 257 (60.9\% del total de pacientes controlados) en el área de Nicoya y 237 (41\%) en la de Desamparados.

\section{Método de recolección}

Se revisaron los expedientes de todos los pacientes diabéticos de los EBAIS estudiados en el primer trimestre de 2001. Se recolectaron en los diabéticos de Nicoya todas las glicemias del año 2000 y sus respectivas Hb1 Ac, con un promedio anual de cada una de las variables por paciente. En los Desamparados se recogió la glicemia más próxima julio de 2000 y concomitantemente su respectiva HbA1c.

\section{Análisis}

a) Se construyeron rangos de los valores de glicemia y de HbA1c, para analizarlos según los criterios de la Asociación Latinoamericana de Diabetes (ALAD; Cuadro1), las guías costarricenses para la atención de pacientes diabéticos en atención primaria (Cuadro 2) y considerar el último valor propuesto por la Asociación Americana de Endocrinología, como meta de buen control: $<6.5 \%{ }^{15,16}$. 
b) Se obtuvieron frecuencias de cada rango de variable y se entrecruzaron para analizar el porcentaje de concordancia (correlación) entre ellas, según la equivalencia glicemia en ayunas y HbA1c, utilizando el esquema del Cuadro $3{ }^{18}$.

$\begin{aligned} & \text { Cuadro 1. Metas para el control de los parámetros de control } \\
& \text { glicémico, a la luz de la evidencia actual según ALAD }\end{aligned}$
\begin{tabular}{lcccc} 
Nivel & Normal & Adecuado & Admisible & Inadecuado \\
\hline $\begin{array}{l}\text { Riesgo complicaciones crónicas } \\
\text { Glicemia ayunas mgs\% }\end{array}$ & $<110$ & bajo & moderado & alto \\
$\begin{array}{l}\text { Glicemia 1-2 horas postprandial } \\
\text { mgs\% }\end{array}$ & $<126$ & $126-140$ & $>140$ \\
HbA1c (\%) & $<6$ & $<180$ & $<180$ & $>180$ \\
\end{tabular}

Fuente: Cita bibliográfica No. 15.

\section{Resultados}

\section{Control glicémico según glicemia en ayunas y HbA1c:}

En los cuadros 4 y 5 se muestra la distribución relativa de glicemias en ayunas y HbA1c en cada zona.

Glicemia en ayunas: el control glicémico analizado según los niveles de glicemia en ayunas, no fue aceptable de acuerdo con los criterios de ALAD en un $57.8 \%$ en zona urbana y un $52.6 \%$ en zona rural, y según las guías nacionales fue malo o crítico en el $67.1 \%$ de los pacientes urbanos y el $61.9 \%$ de los rurales. Solo el $17.9 \%$ de los diabéticos urbanos y el $22.3 \%$ de los rurales estaban en rangos normales de 60-110 mg/dl, mostrando un pobre control metabólico, en ambas zonas de residencia. También el porcentaje en niveles críticos de glicemia fue muy alto, tanto en zona urbana $(35 \%)$ como en la ru$\operatorname{ral}(22.1 \%)$.

HbA1c: Igualmente, el control glicémico analizado según el porcentaje de HbA1c no fue satisfactorio en ninguna de las zonas; no fue aceptable para los criterios de ALAD (> 8\%), en un $57.4 \%$ zona urbana y un $54.4 \%$ en zona rural, y según las guías nacionales fue malo o crítico en el $70 \%$ de la zona urbana y el $67.6 \%$ rural. Solo el $21.1 \%$ de la zona urbana y el $24.1 \%$ de la rural estaban en rangos normales $<6.5 \%$. El nivel crítico fue alto, del $34 \%$ en la urbana y del $40.4 \%$ en la zona rural.

2. Relación de los niveles de glicemia y rangos de equivalencia de HbA1c

Al relacionar las equivalencias entre estos dos parámetros se encontró que en el rango de $60-110 \mathrm{mg} / \mathrm{dl}$, que corresponde a una $\mathrm{HbA} 1 \mathrm{c}<6.5 \%$, solo el $58.5 \%$ de los pacientes urbanos y el $65.3 \%$ de los rurales estaban por debajo de ese nivel; por el contrario, un $22 \%$ de los urbanos y el $10.5 \%$ de los rurales tenían una $\mathrm{HbA} 1 \mathrm{c}>8 \%$. Para el rango de 110-126 $\mathrm{mg} / \mathrm{dl}$, el $30 \%$ urbano y el $37.4 \%$ rural tenían una HbA1c $>8 \%$. Los rangos superiores de glicemia de $>200 \mathrm{mg} / \mathrm{dl}$ presentaron una buena concordancia con $\mathrm{HbA} 1 \mathrm{c}>9.5 \%$, (un $75.6 \%$ en urbanos y un $82.9 \%$ en rurales) (Cuadro 6).

\begin{tabular}{|c|c|c|c|}
\hline \multicolumn{4}{|c|}{$\begin{array}{c}\text { Cuadro 2. Normas de control glicémico } \\
\text { Guías DM Tipo } 2 \text { en Atención Primaria, } \\
\text { Costa Rica, } 2002 .\end{array}$} \\
\hline Nivel & Bueno & Malo & Crítico \\
\hline Glucemia ayunas & $<126$ & $126 \leq 200$ & $>200$ \\
\hline $\begin{array}{l}\text { Glucemia 1-2 horas } \\
\text { pospostprandial }\end{array}$ & $<140$ & $140 \leq 200$ & $>200$ \\
\hline $\mathrm{HbA1c}(\%)$ & $<7$ & $7 \leq 9.5$ & $>9.5$ \\
\hline
\end{tabular}

Fuente: Cita bibliográfica No. 16.

\begin{tabular}{|cc|}
\hline \multicolumn{2}{c|}{$\begin{array}{c}\text { Cuadro 3. Equivalencia de glicemia en ayunas y } \\
\text { HbA1c (18) }\end{array}$} \\
\hline $\begin{array}{c}\text { \% Hemoglobina } \\
\text { glicosilada }\end{array}$ & $\begin{array}{c}\text { Glicemia en } \\
\text { ayunas (mg/dl) }\end{array}$ \\
$<6.5 \%$ & 60 a 110 \\
$6.5<7 \%$ & 111 a 126 \\
$7-7.9 \%$ & 127 a 180 \\
$8-9.5 \%$ & 181 a 200 \\
$>9.5 \%$ & $>200$ \\
\hline
\end{tabular}

Fuente: Cita bibliográfica No. 18.

\section{Discus ión}

La Asociación Americana de Diabetes enfatiza la importancia de asumir la determinación de la HbA1c para el control de las personas con diabetes ${ }^{19}$. Tanto en la DM tipo1 como en la DM tipo 2, se recomienda su determinación cada tres meses, lo que permite medir el éxito terapéutico y realizar ajustes de dosis o añadir nuevas terapias, en caso de que el pobre control así lo indique.

La HbA1c puede ser muy útil para el seguimiento y ajuste de los algoritmos de tratamiento de la DM, favoreciendo un mejor control y cuidado médico, sobre todo en un nivel de atención primaria, en donde se controla la gran mayoría de 
los pacientes ${ }^{20}$.Así, aquellos que sobrepasen el nivel del $8 \%$ deberían ser sometidos a una terapia más intensiva, en procura de lograr beneficios hasta en más del $25-40 \%$ de casos, como demuestra un estudio realizado por Mazze y colaboradores ${ }^{21}$, quienes en un período de meses disminuyeron las cifras de HbA1c del $10.2 \%$ al $8.8 \%$.

\begin{tabular}{|ccccc|}
\hline $\begin{array}{c}\text { Cuadro 4. Distribución total y porcentual de la } \\
\text { glicemia de los pacientes diabéticos, según } \\
\text { zona de residencia. }\end{array}$ \\
$\begin{array}{c}\text { Glicemia en ayunas } \\
\text { (mg/dl) }\end{array}$ & Urbano & \multicolumn{2}{c|}{ Rural } \\
\hline Intérvalos & $\mathrm{N}$ & $\%$ & $\mathrm{~N}$ & $\%$ \\
\hline 60 a 110 & 41 & 17.3 & 71 & 27.6 \\
111 a 126 & 37 & 15.6 & 27 & 10.5 \\
127 a 180 & 70 & 29.6 & 83 & 32.3 \\
181 a 200 & 6 & 2.5 & 19 & 7.5 \\
$>200$ & 83 & 35 & 57 & 22.1 \\
\hline Total & 237 & 100 & 257 & 100 \\
\hline
\end{tabular}

\begin{tabular}{|c|c|c|c|c|}
\hline \multicolumn{5}{|c|}{$\begin{array}{l}\text { Cuadro 5: Distribución total y porcentual de los } \\
\text { niveles de HbA1c, según zona de residencia }\end{array}$} \\
\hline $\mathrm{HB} 1 \mathrm{aC}(\%)$ & \multicolumn{2}{|c|}{ Urbano } & \multicolumn{2}{|c|}{ Rural } \\
\hline Intérvalos & $\mathrm{N}$ & $\%$ & $\mathrm{~N}$ & $\%$ \\
\hline$<6.5 \%$ & 50 & 21.1 & 62 & 24.1 \\
\hline $6.5<7 \%$ & 21 & 8.9 & 21 & 8.0 \\
\hline $7-7.9 \%$ & 30 & 12.6 & 34 & 13.2 \\
\hline $8-9.5 \%$ & 54 & 22.8 & 36 & 14.0 \\
\hline$>9.5 \%$ & 82 & 34.6 & 104 & 40.4 \\
\hline Total & 237 & 100 & 257 & 100 \\
\hline
\end{tabular}

\begin{tabular}{|c|c|c|c|c|c|c|c|c|c|c|}
\hline \multirow{3}{*}{$\begin{array}{c}\text { Nivel de } \\
\text { glicemia }(\mathrm{mg} / \mathrm{dl})\end{array}$} & \multicolumn{10}{|c|}{$\mathrm{HbA1c}$} \\
\hline & \multicolumn{2}{|c|}{$<6.5 \%$} & \multicolumn{2}{|c|}{$6.5<7 \%$} & \multicolumn{2}{|c|}{$7-8 \%$} & \multicolumn{2}{|c|}{$>8-9.5 \%$} & \multicolumn{2}{|c|}{$>9.5 \%$} \\
\hline & U & $\mathrm{R}$ & U & $\mathrm{R}$ & U & $\mathrm{R}$ & U & $\mathrm{R}$ & U & $\mathrm{R}$ \\
\hline 60 a 110 & 58.5 & 65.3 & 9.8 & 12 & 9.8 & 12 & 12.2 & 8 & 9.8 & 2.5 \\
\hline 111 a 126 & 32.4 & 20.8 & 29.7 & 8.3 & 10.8 & 33.3 & 24.3 & 20.8 & 2.7 & 16.6 \\
\hline 127 a 180 & 20.8 & 13 & 8.9 & 11.2 & 28.4 & 15.1 & 20.2 & 21.9 & 21.8 & 38.6 \\
\hline 181 a 200 & - & 5.2 & - & - & 16.7 & 1.0 & 50.0 & 15.2 & 33.3 & 78.9 \\
\hline$>200$ & - & - & .9 & - & 1.7 & 2 & 21.9 & 15.2 & 75.6 & 82.9 \\
\hline
\end{tabular}

El estudio indica, tanto en zona urbana como rural, diferencias importantes entre los niveles de glicemia en ayunas y los de hemoglobina glicosilada reportados, particularmente en las cifras inferiores de glicemia en ayunas ( $<126 \mathrm{mg} / \mathrm{dl})$, en donde el médico tratante no ajusta el tratamiento por considerarlo un buen control. El $49 \%$ de los pacientes de la zona urbana y el $47.9 \%$ de la zona rural, que obtuvieron glicemias en ayunas $<126 \mathrm{mg} / \mathrm{dl}$, manejaban cifras de HB1Ac $>8 \%$. Si hubieran sido valorados solo a la luz de la glicemia, no se hubieran visto favorecidos con un cambio e intensificación de su terapéutica, persistiendo el consecuente daño de órganos blancos, por las hiperglicemias no documentadas en los tres meses anteriores a su control. Más aún, el $12.5 \%$ de los paciente urbanos y el $19.1 \%$ de los rurales con glicemias en ayunas en rangos ideales (<110mg \%), tenían hemoglobinas glicosiladas en los rangos considerados "críticos" (> 9.5\%).

Basarse en la determinación de la glicemia en ayunas en los pacientes diabéticos, cada vez que acuden a sus citas control con el fin de monitorear su control glicémico, no es confiable, como lo demuestran los datos del estudio. Este examen puede estar influido negativamente por una serie de factores que tienen que ver con la adherencia del paciente y la calidad de su control por los equipos de salud. La determinación de $\mathrm{HbA} 1 \mathrm{c}$ es, por lo tanto, mandatorio e imprescindible.

A pesar del beneficio incuestionable de la HbA1c, se conoce, por ejemplo, que en los Estados Unidos de Norteamérica el 37\% de los diabéticos tipo 2 tienen niveles superiores al $8 \%{ }^{22}$, y se afirma que este examen se subutiliza, pues apenas se le practica al $50 \%$ de los diabéticos tipo $2^{23}$.

En Costa Rica se desconoce el porcentaje de los diabéticos que esta siendo controlado con la HbA1c, pero definitivamente en atención primaria, en donde se maneja la mayoría de ellos, no están siendo controlados así, o ya sea porque la prueba no está asequible al primer nivel de atención, o porque los médicos no la utilizan pese a que exista, como ocurre en el área de salud de Nicoya.

En el Plan de Atención a la Salud de las personas 2001-2006 de la $\mathrm{CSSS}^{24}$, se contempla que para 2004 esta prueba se utilice en todos los niveles de atención de la CCSS, pero hay varios impedimentos para que se implemente. Una de las razones es que se trata de una prueba sumamente cara si se realiza con técnicas adquiridas en el mercado, con una relación contractual de las áreas de salud como entidades independientes y las empresas privadas suplidoras de las técnicas y de los reactivos. Es necesario, considerando los recursos económicos del país, que se estandarice la misma técnica a nivel nacional, con el fin de comprar los reactivos en forma centralizada y así abaratar los costos. Además, 
el uso de una sola técnica permitiría comparar los resultados del control glicémico de todos los pacientes diabéticos del país, independientemente de su lugar de residencia, como parte del monitoreo en vigilancia epidemiológica de la DM, patología que ocupa el primer lugar en costos hospitalarios.

Uniformar la técnica de la determinación de la HB1Ac es necesario y posible en un país tan pequeño como Costa Rica, con una infraestructura, que hace factible su implementación.

El grado de control glicémico mostrado en los dos grupos de diabéticos, a pesar de provenir de regiones geográficas y socioeconómicas diferentes, no es muy disímil, y en ambos se muestra una población muy importante de pacientes en mal control. En zona urbana el 70\%, y en la rural el 67.6\%, estaban mal controlados, según la normativa de las Guías de Costa Rica, y tenían un control inadecuado de un $57.4 \%$ en zona urbana y un $54.4 \%$ en zona rural de acuerdo con la de ALAD.

Lo anterior obliga a establecer estrategias para el mejoramiento de la calidad del control en el primer nivel de atención de las áreas de salud de los EBAIS analizados, y provoca una llamada de alerta para considerar situaciones similares en otras áreas de salud costarricenses.

Para el manejo de estos pacientes mal controlados es indispensable utilizar un enfoque de riesgo, y lo que requiere una buena coordinación entre los interniveles de atención, según la complejidad. Cifras de HbA1c en niveles críticos o cifras persistentemente $>8 \%$, a pesar de cambios en el tratamiento, podrían servir como indicadores de riesgo. La HbA1c debería convertirse en una de las medidas para valorar riesgo y tener criterios acerca del uso de la referencia a niveles de mayor complejidad de atención, ya sea al nivel II a Medicina Interna, o al nivel III cuando se acompañe de complicaciones crónicas que no se puedan resolver en el II nivel.

Los resultados permiten concluir que la hemoglobina glicosilada es el patrón de oro para el control del paciente diabético. Si esta fuera determinada en todo el Sistema de Salud de Costa Rica, sus niveles reflejarían la calidad del sistema, y permitirían establecer comparaciones entre pacientes y la severidad de la condición entre las diferentes regiones geográficas y áreas de salud. Los valores superiores al $8 \%$ probarían la insuficiencia de la intervención en los grupos estudiados al igual que el estatus de cada paciente. Su implementación, tanto en disponibilidad como con una técnica única, debe ser obligatoria en todas la áreas de salud de Costa Rica, y la meta debe ser que todos los pacientes diabéticos tengan un nivel de $\mathrm{HbA} 1 \mathrm{c}$ menor al $6.5 \%$.
Abstract

Introduction: Glycosilated hemoglobin (HbA1c) is at present the best available test to reflect the metabolic control of diabetic patients, however its use is not yet systematic in our national health system.

Objective: To determine the quality of metabolic control of Costa Rican diabetics by urbanization level, according to fasting blood glucose or HbA1c and to compare their respective equivalent values.

Study design and methods: Retrospective study comparing the two parameters mentioned above, 237 diabetics controlled at 6 Primary Health Care centers (EBAIS) of an urban Health Area (Desamparados, one county of the San José province), and 257 diabetics controlled in 3 Primary Health Care Centers (EBAIS) of a rural Health Area, (Nicoya, one county of the province of Guanacaste), during the year 2000. In the patients of Desamparados the blood glucose closest to the month of July of 2000 and its respective HbA1c, taken the same day, was registered. In those diabetics from Nicoya the average blood glucose and HbA1c of the year 2000 was used for the study.

Results: Fasting blood glucose in the urban diabetics was more frequently at higher levels: $35 \%>200 \mathrm{mg} / \mathrm{dl}, 20.5 \%$ between $141-180 \mathrm{mg} / \mathrm{dl}$ and only $17.9 \%$ were within normal ranges $(60-110 \mathrm{mg} / \mathrm{dl})$, thus exhibiting poor metabolic control. Diabetics from the rural area had similar results, with $27 \%$ over $200 \mathrm{mg} / \mathrm{dl}, 22.9 \%$ in the ranges of $141-180$ and $22.3 \%$ within normal ranges.

The HbA1c results were not adequate either, at the urban setting, $34.6 \%$ were over the critical values of $9.5 \%$, and only $21.1 \%$ were lower than $6.5 \%$, compared to $40.4 \%$ and $14.0 \%$, respectively in the rural area.

When the equivalent ranges of fasting blood glucose were compared with $\mathrm{HbA1c}$, big differences were found; for normal glycemic ranges $(60-110 \mathrm{mg} / \mathrm{dl})$, only $58.5 \%$ of the urban data, and $65.3 \%$ of the rurals, coincided with its equivalent of HbA1c $(<6.5 \%) ; 30 \%$ of the urban patients and $37 \%$ rural patients had for this same values, HbA1c over $8 \%$. When the values of blood glucose were over $200 \mathrm{mg} / \mathrm{dl}$, the relation with its $\mathrm{HbA} 1 \mathrm{c}$ equivalent range (> 9.5\%) was high: $75.6 \%$ in urban area and $82.9 \%$ in the rural area.

Conclusion: glycosilated hemoglobin is the best method to evaluate the quality of metabolic controlin diabetic patients, especially in those that handle fasting levels $<200 \mathrm{mg} / \mathrm{dl}$. In Costa Rica glycosilated hemoglobin determination has not been generalized at primary health level and too many patients are not receiving the benefit of an intensive therapy, because they are being evaluated only with fasting blood glucose. The implementation of glycosilated hemoglobin is posible for our national health system and it should be compulsory in all Health Areas of Costa Rica. 


\section{Referencias}

1. Conget I. Diagnóstico, clasificación y patogenia de la diabetes mellitus. Rev Esp Cardiol $2002 ; 55$ : 528-35.

2. American Public Health Association. Diabetes. En United Book Press Ed. Chronic Disease Epidemiology and Control Washington DC, USA, 1998 : Chapter 14: 421.

3. Anónimo. El Proyecto de Reforma del Sector Salud. Acta Med Costarric 1993 ; 36: 17-23.

4. Organización Panamericana de la Salud. Estadísticas vitales de salud de Costa Rica. www.paho.org/Spanish/SHA/prflcor.htm.

5. The UK Prospective Diabetes Study (UKPDS) Group. Intensive blood-glucose control with sulphonylureas or insulin compared with conventional treatment and risk of complication in patients with type 2 diabetes (UKPDS 33). Lancet 1998; 352: 837-853.

6. The Diabetes Control and Complications Trial Research Group. The effect of intensive treatment of diabetes on the development and progression of long-term complications in insulin-dependent diabetes mellitus. N Eng J Med 1993; 329: 977-986.

7. Ohkubo Y, Kishikawa H, Araki E, Miyata T, Isami S, Motoyoshi S, Kojima Y, Furuyoshi N y Shichiri M. Intensive insulin therapy prevents the progression of diabetic microvascular complications in Japanese patients with non-insulin dependent diabetes mellitus : a randomized prospective 6 year study. Diabetes Res Clin Pract 1995; 28: 103-117.

8. Klein R. Hyperglycemia and microvascular and macrovascular disease. Diabetes Care 1995 ; 18: 258-268.

9. The Diabetes Control and Complications Trial Research Group. The relationship of glycemic exposure (HbA1c) to the risk of development and progression of retinopathy in the Diabetes Control and Complications Trial. Diabetes 1995 ; 44: 968-983.

10. Mathew C. Riddle, MD y Dianne M. Karl, HbA1c: La mejor forma de medir la calidad del tratamiento. Clinical Diabetes 1996; :79-82.

11. Jiménez MF y Ruíz L. Niveles de glicemia y de hemoglobina glicosilada en un grupo de diabéticos nicoyanos. Acta Med Costarric 2001; 43 (suplemento 1): 15.

12. Laclé A. Perfil del paciente diabético tipo 2 de 55 y más años, de una clínica periférica de la Caja Costarricense de Seguro Social. Acta Med Costarric 1999; 41:46-51.
13. Balma A. Diabetes Mellitus tipo II en la Clínica de Esparza. Medicina, Vida y Salud 2002; 5:18.

14. Uiversidad de Costa Rica. Información general de Costa Rica. http://www.sibdi.bldt.ucr.ac.cr

15. Asociación Latinoamericana de Diabetes (ALAD). Guías ALAD 2002. www.alad.org/docs.html,

16. Caja Costarricense de Seguro Social. Departamento de Medicina Preventiva. Manual para la Atención Integral de la Diabetes Mellitus en el Primer Nivel de Atención (con énfasis en la DM tipo 2). Unidad de Imprenta CCSS. Costa Rica, 2002.

17. American Association of Clinical Endocrinologists and the American College of Endocrinology. The American Association of Clinical Endocrinologists Medical Guidelines for the Management of Diabetes Mellitus:The AACE System of Intensive Diabetes Self-Management2002 Update. Endocrine Practice 2002; 8 (Suppl.1) : 41-65.

18. Padilla G, Aráuz AG y Sánchez G. Guía para la enseñanza en diabetes mellitus: primer nivel de atención en salud. Imprenta INCIENSA. Tres Ríos, Cartago, Costa Rica, 2002.

19. American Diabetes Association. Standards of Medical Care for Patients with Diabetes Mellitus. Diabetes Care 2002; 25 (supplement 1): S37.

20. Peters AL, Davidson MB, Schriger DL, Hasselblad V. A clinical approach for the diagnosis of diabetes mellitus: an analysis using glycosylated hemoglobin levels. Meta-analysis Research Group on the Diagnosis of Diabetes Using Glycated Hemoglobin Levels. JAMA1996; 276 : 1246-1252.

21. Mazze RS, Etzwiler DD, Strock E, Peterson K, McClave CR 2nd, Meszaros JF, et al Staged diabetes management. Toward an integrated model of diabetes care. Diabetes Care 1994(Suppl 1); 17: 56-66.

22. Harris MI, Eastman RC, Cowie CC, Flegal KM, Eberhardt MS. Racial and ethnic differences in glycemic control of adults with type 2 diabetes. Diabetes Care 1999 ; 22: 403-408.

23. Clark CM, Fradkin JE, Hiss RG, Lorenz RA, Vinicor F, Warren-Boulton E. Promoting Early Diagnosis and Treatment of Type 2 Diabetes. The National Diabetes Education Program. JAMA 2000; 284 : 363365 .

24. Caja Costarricense de Seguro Social. Plan de Atención a la Salud de las Personas. Corporación Litográfica Internacional S.A. San José, Costa Rica, 2002. Páginas 95 a 100. 